\title{
Genetic variability of milk components based on mid-infrared spectral data
}

\author{
H. Soyeurt, ${ }^{*} \dagger^{1}$ I. Misztal, $\ddagger$ and N. Gengler ${ }^{*} \dagger$ \\ *Animal Science Unit, Gembloux Agro-Bio Tech, University of Liège, 5030 Gembloux, Belgium \\ †National Fund for Scientific Research, 1000 Brussels, Belgium \\ $\ddagger$ Department of Animal and Dairy Science, University of Georgia, Athens 30605
}

\begin{abstract}
The aim of this study was to estimate the genetic parameters of the mid-infrared (MIR) milk spectrum represented by 1,060 data points per sample. The dimensionality of traits was reduced by principal components analysis. Therefore, 46 principal components describing $99.03 \%$ of the phenotypic variability were used to create 46 new traits. Variance components were estimated using canonical transformation. Heritability ranged from 0 to 0.35 . Twenty-five out of 46 studied traits showed a permanent environment variance greater than genetic variance. Eight traits showed heritability greater than 0.10. Variances of original spectral traits were obtained by back transformation. Heritabilities for each spectral data points ranged from 0.003 to 0.42 . In particular, 3 MIR regions showing moderate to high heritability estimates were of potential genetic interest. Heritabilities for specific wave numbers, linked with common milk traits (e.g., lipids, lactose), were similar to those estimated for these traits. This research confirms the genetic variability of the MIR milk spectrum and, therefore, the genetic variation of milk components. The objective of this study was to better understand the genetics of milk composition and, maybe in the future, to select animals to improve milk quality.
\end{abstract}

Key words: mid-infrared, milk, principal component, heritability

\section{INTRODUCTION}

Milk produced by dairy cows is a complex combination of at least 10,000 different biomolecules (Jelen, 2007). However, only a few nonspecific milk components (e.g., fat, protein, and lactose) are included in the current animal selection programs. Therefore, the genetic variability of a large part of the detailed milk composition is unknown, especially because the chemi-

Received August 3, 2009.

Accepted November 23, 2009.

${ }^{1}$ Corresponding author: hsoyeurt@ulg.ac.be cal reference analysis used to measure these specific components is expensive.

Mid-infrared (MIR) spectrometry is a powerful method routinely used through the world to evaluate milk composition and different food products such as milk (e.g., Sivakesava and Irudayaraj, 2002; Soyeurt et al., 2006) and cheese (e.g., Karoui et al., 2006). Being quick and inexpensive, MIR spectrometry is used in daily milk recording to predict the contents of major components used in current selection programs, such as the percentages of fat and protein. These traits are predicted from different calibration equations applied to the spectral data generated by the MIR spectrometer during the infrared analysis of milk. This spectral data results from the interaction of the milk molecules with the MIR laser (William and Norris, 2001). Consequently, the MIR spectrum reflects the global composition of milk, but this information is currently underused. Theoretically, the MIR data should reflect the genetic variation in milk components, so it could be interesting to study directly the spectral information as a way to study the expression of genes linked to the detailed milk composition of dairy cows.

The MIR spectrum of cow milk provided by the MilkoScan FT6000 spectrometer (Foss, Hillerød, Denmark) contains 1,060 data points, also named pin numbers, and is expressed in transmittance. The number of estimated parameters grows as a quadratic function of the number of traits considered. Therefore, the estimation of variance components for more than 1,000 traits using the current methods used in quantitative genetics, such as REML (Searle et al., 1992), is a challenge. Misztal (2008) mentioned that the limits of REML methodologies could be around 200,000 animals, 2 million equations, and 5 traits. Even if progress in computers is becoming steadily available, the computational resources for the simultaneous estimation of variance components for each spectral trait using multitrait REML is not possible without reducing the dimensionality of traits. Current methods used in quantitative genetics to resume the information use data transformation techniques, rendering the multitrait systems to multiple single-trait analysis (Misztal et al., 1995). 
The objective of this study was to analyze MIR milk spectral data and to estimate genetic parameters using trait reduction techniques and multitrait REML.

\section{MATERIALS AND METHODS}

\section{Spectral Data and Animal Population}

During the routine milk recording in the Walloon part of Belgium (managed by the Walloon Breeding Association, Ciney, Belgium), a total of 7,621 milk samples were collected from 1,594 first-parity Holstein cows ( $>84 \%$ of Holstein gene) from April 2005 to December 2007 in 92 herds. It represents 4.78 records per cow, with a minimum value equal to 1 and a maximum value equal to 12 . All samples were collected between 5 and 365 DIM. Milk Committee (Battice, Belgium) analyzed the samples using a Milkoscan FT6000 MIR spectrometer (Foss). Major milk components such as fat and protein were quantified by the application of specific calibration equations on generated MIR spectral data. Traditionally, these spectral data are directly erased after analysis. The new approach of this study was to record the MIR spectra represented by 1,060 data points before the deletion. The complete pedigree of the cows with records was extracted and included 7,326 animals.

\section{Trait Reduction}

Principal component analysis (PCA) was applied to the collected spectral data to decrease the number of traits analyzed simultaneously. The purpose of PCA is to derive the smallest number of linear combinations (principal components; PC) from a set of new traits that retain the largest amount of information contained in the original traits (Palm, 1998).

If $\mathbf{V}$ represents the phenotypic (co)variances matrix for the 1,060 initial spectral traits for daily test records, the transformation from the eigenvalue decomposition can be derived as $\mathbf{V}=\mathbf{U D U}$, where $\mathbf{U}$ corresponds to the eigenvectors matrix and $\mathbf{D}$ is the diagonal matrix of eigenvalues. Using PROC PRINCOMP in SAS (SAS Institute, 1994), PCA was applied to the spectral data. The PC were chosen based on the importance of information given by these parameters.

New traits were defined as functions of the eigenvectors associated with the largest eigenvalues. The new traits were obtained as

$$
\mathbf{y}_{U}=\left(\mathbf{I}_{n} \otimes \mathbf{U}_{R}^{\prime}\right) \mathbf{y},
$$

where $\mathbf{U}_{R}$ is the reduced matrix containing the chosen eigenvectors, $\mathbf{I}_{n}$ is an identity matrix of dimension $n$ equal to the number of records, and $\mathbf{y}$ is the vector including the 1,060 original traits, sorted within records.

\section{Model and Estimation of (Co)variances}

New traits defined from PCA were analyzed using multiple diagonalization (Misztal et al., 1995). This strategy allows the recovery of genetic and nongenetic (co)variances among PCA traits using a transformation matrix $\mathbf{T}$. The final transformation can be written as

$$
\mathbf{y}^{*}=\left(\mathbf{I}_{n} \otimes \mathbf{T}\right) \mathbf{y}_{U}=\left(\mathbf{I}_{n} \otimes \mathbf{T} \mathbf{U}_{R}^{\prime}\right) \mathbf{y}=\left(\mathbf{I}_{n} \otimes \mathbf{Q}\right) \mathbf{y},
$$

where $\mathbf{y}^{*}$ is the vector including the new traits derived from $\mathrm{PCA}$, and $\mathbf{Q}=\mathbf{T} \mathbf{U}_{R}{ }^{\prime}$ represents the final transformation matrix. The transformed traits are linear combinations of the initial traits with uncorrelated genetic, permanent environment, and residual (co)variance matrices.

The multitrait mixed model used to estimate the (co) variance components can be written as

$$
\left(\mathbf{I}_{n} \otimes \mathbf{Q}\right) \mathbf{y}=\mathbf{y}^{*}=\mathbf{X} \boldsymbol{\beta}^{*}+\mathbf{Z} \mathbf{u}^{*}+\mathbf{Z} \mathbf{p}^{*}+\mathbf{e}^{*},
$$

where $\mathbf{y}^{*}$ is the vector of transformed test-day spectral traits; $\boldsymbol{\beta}^{*}$ is the vector of fixed effects (herd $\times$ test date, stage of lactation defined as 20 equilibrated classes covering an average of 15 DIM); $\mathbf{p}^{*}$ is the vector of permanent environmental random effects; $\mathbf{u}^{*}$ is the vector of additive genetic animal effects; $\mathbf{X}$ and $\mathbf{Z}$ are incidence matrices; and $\mathbf{e}^{*}$ is the vector of random residual effects.

The variance components were estimated using the MD-EM-REML program developed by Misztal (1994). The distribution of random effects was assumed to be normal. Estimated (co)variances on the transformed scales were back transformed toward the original 1,060 spectral traits using a 2-step approach: 1) back transforming to PCA traits, and 2) back transforming to original spectral traits.

\section{Standard Error of Estimates}

Standard errors of the estimated variance components and heritability values can be derived from the inverse of the negative average information matrix, which is an approximation of the asymptotic dispersion matrix. The EM-REML algorithm does not provide this matrix (Misztal, 2008). The following strategy was implemented to obtain an approximation of standard errors: the transformation matrix generated by this algorithm was used to transform the dependant traits 


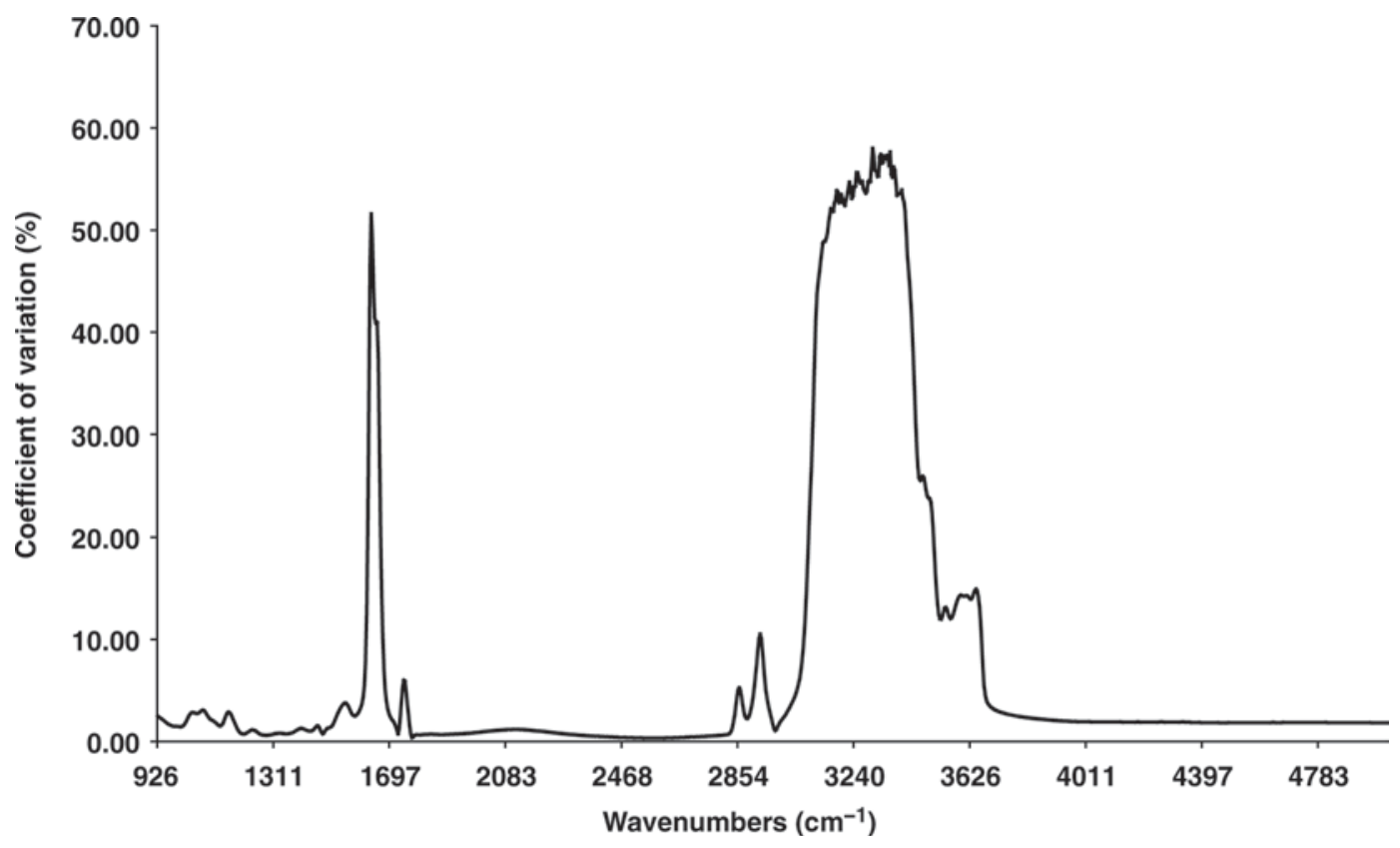

Figure 1. Coefficients of variation calculated for the 1,060 spectral data points.

into independent traits. The same procedure was done to convert the variances obtained by MD-EM-REML. The AI-REML algorithm permits the calculation of the approximate SE (Misztal, 2008). Therefore, additional AI-REML rounds were performed on every single independent transformed trait, and the standard errors for the PCA traits were approximated by a back transformation.

\section{RESULTS}

\section{PCA Applied to 1,060 Data Points}

The phenotypic variability was first studied from the coefficients of variation. Figure 1 illustrates these coefficient values, defined as the ratio of standard deviation to the mean multiplied by 100 and calculated for each spectral data point. Two MIR regions showed coefficients of variation of more than $30 \%$. These regions were located between $1,628 \mathrm{~cm}^{-1}$ and $1,658 \mathrm{~cm}^{-1}$ and between $3,105 \mathrm{~cm}^{-1}$ and $3,444 \mathrm{~cm}^{-1}$ and represented a total of 98 spectral data points.

Table 1 presents the percentages described by the first $46 \mathrm{PC}$, the estimates, and the standard error of variances ratios (in function of the phenotypic variance) estimated for the genetic, permanent environmental, and residual effects. The PCA traits were sorted according to their relative eigenvalues. The part of the spectral information explained by each PC decreased rapidly. Therefore, $46 \mathrm{PC}$ that described $99.03 \%$ of spectral variability were chosen in this study. The limitation at $99 \%$ was chosen arbitrarily and was assumed to be sufficient in the first part of this study to estimate the heritability of milk MIR spectral data. A part of the spectral variability is not related to the milk (see more details in the Discussion section). Therefore, a second selection of PC is presented in the second part of this study.

Forty-six new traits were created from these 46 selected eigenvectors and transformed into uncorrelated traits. Heritability values for these new traits ranged between 0.00 and 0.35 . Eight out of 46 studied traits showed heritability superior to 0.10 (Table 1). Variances ratio for the permanent environment ranged from 0.00 to 0.20 . Variance ratio for the permanent environment of 25 out of 46 studied traits was greater than the heritability estimated for these same traits. Residual variance ratio ranged from 0.46 to 0.98 . For all traits, the approximate standard errors were small in all studied variance ratios. However, these values could be underestimated by the methodology used.

The rankings of $\mathrm{PC}$ as a function of phenotypic or genetic effect were different (Table 1). For instance, the second PC was not the second PC showing the highest heritability value. The same observation can be made for the 2 other random effects.

The ratio of the sum of the genetic and permanent environmental variances to the total variance was calculated to present the repeatability of the MIR milk spectrum. The repeatability as well as heritabilities for the 1,060 spectral data points were estimated after the 
Table 1. Estimates and SE of variance ratios for genetic, permanent environmental, and residual random effects calculated for the 46 traits estimated from the 46 principal components (PC)

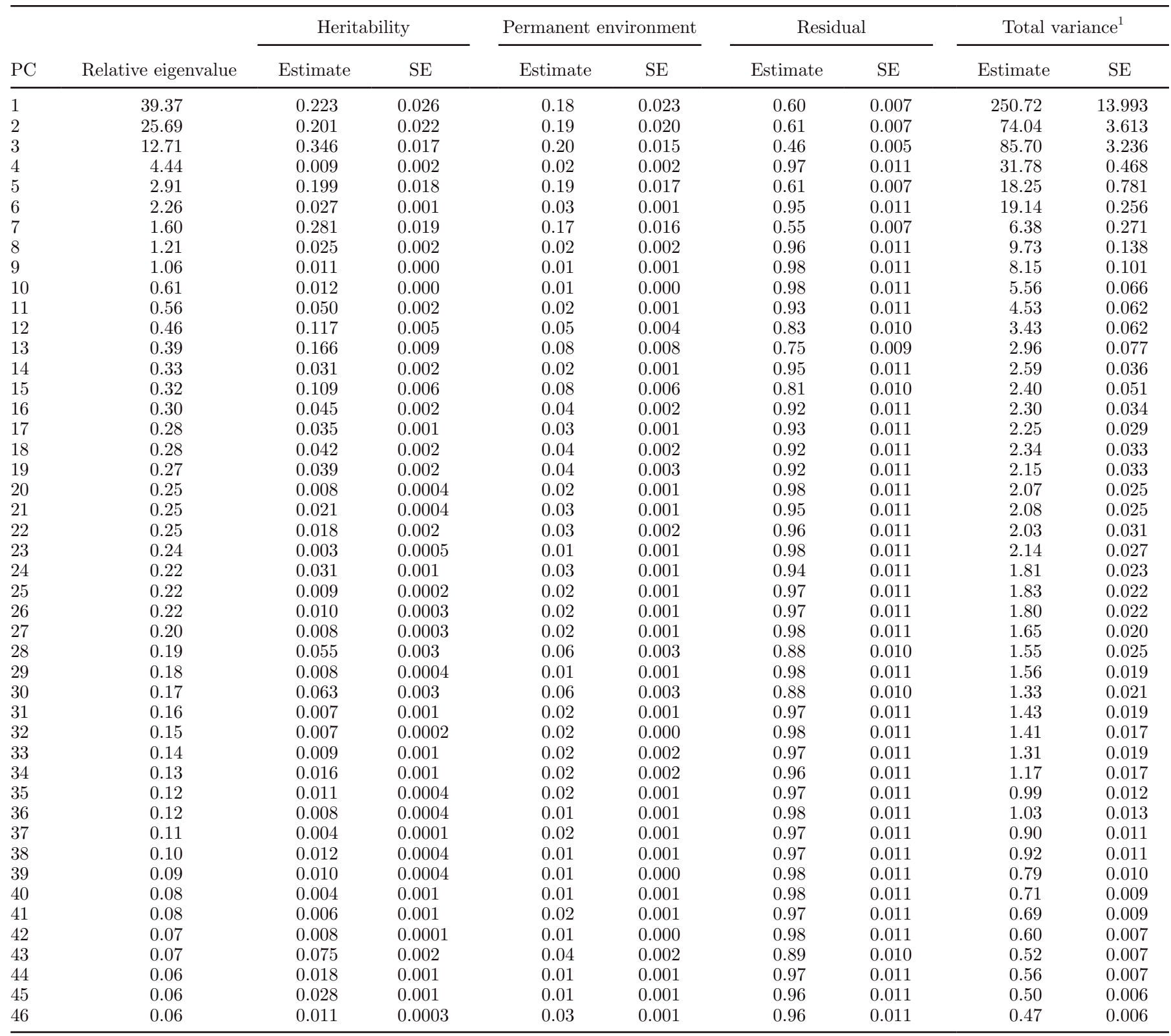

${ }^{1}$ The total variance is expressed in transmittance squared; the transmittance is the unit of the spectral data.

inverse transformation. Figure 2 illustrates the repeatability and the heritability values across the MIR milk spectrum. In the same figure, the spectrum has also been represented to show the MIR milk spectrum regions with the highest heritability, or repeatability, or both. The ratio of the sum of the genetic and permanent environmental variances to the total variance ranged from 0.01 to 0.64 . The range of heritability values was more limited. Heritability for the spectral data points ranged from 0.00 to 0.42 . The trends observed in Figure
2 for the repeatability and heritability estimates had the same pattern. Figure 2 clearly shows 3 regions with moderate to high genetic variability. By choosing arbitrarily a threshold of heritability value equal to 0.10 , 3 regions can be isolated and are located between 926 and $1,612 \mathrm{~cm}^{-1}, 1,682$ and $3,062 \mathrm{~cm}^{-1}$, and 3,672 and $5,010 \mathrm{~cm}^{-1}$. These MIR regions with moderate to high heritability estimates included only 886 spectral data points instead of the 1,060 spectral points ( $85 \%$ of the total spectral points). 
Table 2. Estimates and SE of variance ratios for genetic, permanent environmental, and residual random effects calculated for the 8 traits estimated from the 8 principal components (PC)

\begin{tabular}{|c|c|c|c|c|c|c|c|c|c|}
\hline \multirow[b]{2}{*}{$\mathrm{PC}$} & \multirow[b]{2}{*}{ Relative eigenvalue } & \multicolumn{2}{|c|}{ Heritability } & \multicolumn{2}{|c|}{ Permanent environment } & \multicolumn{2}{|c|}{ Residual } & \multicolumn{2}{|c|}{ Total variance $^{1}$} \\
\hline & & Estimate & $\mathrm{SE}$ & Estimate & $\mathrm{SE}$ & Estimate & $\mathrm{SE}$ & Estimate & $\mathrm{SE}$ \\
\hline 2 & 30.55 & 0.11 & 0.020 & 0.24 & 0.021 & 0.65 & 0.008 & 65.01 & 3.225 \\
\hline 3 & 15.16 & 0.25 & 0.033 & 0.26 & 0.031 & 0.50 & 0.006 & 77.86 & 5.410 \\
\hline 4 & 3.39 & 0.21 & 0.029 & 0.21 & 0.027 & 0.57 & 0.008 & 17.13 & 1.104 \\
\hline 5 & 1.81 & 0.34 & 0.026 & 0.18 & 0.021 & 0.48 & 0.006 & 5.54 & 0.292 \\
\hline 8 & 0.30 & 0.17 & 0.025 & 0.17 & 0.023 & 0.66 & 0.009 & 1.26 & 0.072 \\
\hline
\end{tabular}

${ }^{1}$ The total variance is expressed in transmittance squared; the transmittance is the unit of the spectral data.

\section{PCA Applied to 886 Data Points}

These results suggest that some PC did not show a genetic interest. For the example, it was decided arbitrarily to operate a second selection based on the MIR regions with heritability values superior to 0.10 . Aforementioned, these regions represented 898 spectral data points. The methodology followed was the same as the one presented in the first part of this study. Eight new variables were defined because the first $8 \mathrm{PC}$ described $99.18 \%$ of the initial spectral information. Because the number of studied traits was not too large, the SE and variances were estimated directly by AI-REML. Heritability values for the 8 studied new traits ranged between 0.10 and 0.35 (Table 2). Variance ratios for the permanent environment ranged from 0.13 to 0.26 . The first $2 \mathrm{PC}$ with the highest eigenvalues were not those $\mathrm{PC}$ with the highest heritabilities.

\section{DISCUSSION}

The rapid decrease of spectral variability described by PC (Figure 1) could be explained by the strong link existing among several spectral data points. Correla-

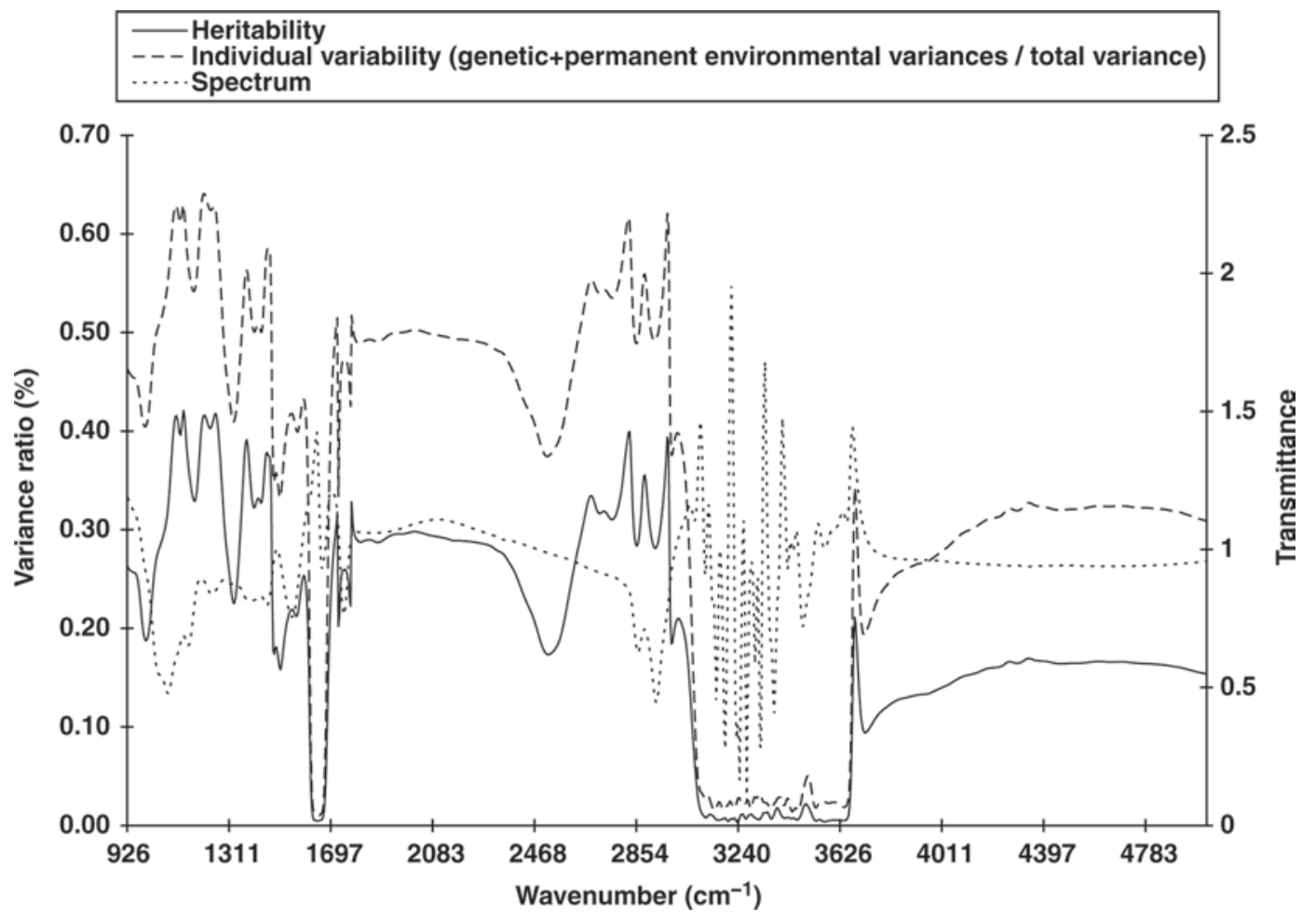

Figure 2. Repeatability estimated from the ratio of the sum of the genetic and permanent environmental variances to the total variance and heritability calculated for 1,060 spectral data points expressed in wavenumber $\left(\mathrm{cm}^{-1}\right)$ and illustration of mid-infrared milk spectrum. 
tions estimated for few adjacent spectral points showed high values (data not shown).

The moderate heritability values observed for 8 studied traits (Table 1 ) confirmed the genetic variability of the milk MIR spectrum. However, not all MIR regions showed sufficient genetic variance and repeatability to involve a real interest for animal selection. The evolution across MIR spectral data of the ratio of the sum of the genetic and environmental variances to the total variance showed the same pattern as the one observed for the heritability values.

The very low heritabilities of 2 specific regions located between 1,616 and $1,678 \mathrm{~cm}^{-1}$ and between 3,066 and $3,668 \mathrm{~cm}^{-1}$ (Figure 2) could reflect the laboratory environment, or the internal parameters of the MIR spectrometer, or both. A contribution of water around $3,420 \mathrm{~cm}^{-1}$ was observed by Karoui et al. (2006). This difference in heritability estimates could be previously deduced from the values of the variation coefficient presented in Figure 1. The coefficients of variation were high for the specific regions with low heritability estimates. Therefore, these 2 regions showed a phenotypic variation largely superior to the rest of the spectrum. The high residual variances ratios for the traits with low heritability (Table 1) confirmed that some MIR regions were less important for milk composition. Moreover, the first $2 \mathrm{PC}$ estimated from the second selection of MIR spectral data and showing the highest eigenvalues (Table 2) did not present the highest values of heritability. It suggests that most variability of the spectral information is not caused by genetics. However, the advantage of a genetic selection is its additive aspect.

The interpretation of individual variability, or genetic variability, or both, of MIR spectrum is difficult because milk represents a combination of a large number of different molecules. However, the heritability for some wave numbers can be interpreted. The high values of heritability observed were consistent with previous knowledge, especially for the MIR region located between 926 and $1,616 \mathrm{~cm}^{-1}$ (Figure 2). This region is called the fingerprint region, referring to $\mathrm{C}-\mathrm{O}$ and $\mathrm{C}-\mathrm{C}$ stretching modes (1,153-900 $\mathrm{cm}^{-1}$; Karoui et al., 2006). In fact, these chemical bonds are common in the chemical structure of the major components of milk such as, for example, sugars, fats, and protein. Therefore, these spectral data provide a direct indication of the quantity of major components contained in the analyzed milk sample. In other words, it is the identity card of the milk sample. The high heritability estimates observed between 2,800 and $3,000 \mathrm{~cm}^{-1}$ could be related to the content of lipids in cow milk (Figure 2). In fact, the vibrations of ester linkage and $\mathrm{C}-\mathrm{H}$ stretch groups related to fatty acids are assumed to occur in this region (Si- vakesava and Irudayaraj, 2002). This one is dominated by 2 strong bands at 2,846 and 2,915 $\mathrm{cm}^{-1}$ (Dufour et al., 2000). The average of heritability for the data points located between 2,800 and $3,000 \mathrm{~cm}^{-1}$ was 0.31 . This value was close to the heritability for the percentage of fat in milk. Welper and Freeman (1992) found 0.41 as heritability for fat percentage. Ikonen et al. (1999) estimated the heritability of fat percentage equal to 0.43. More recently, Miglior et al. (2007) found larger heritability value (0.55) for first-parity Canadian Holstein cows. Sivakesava and Irudayaraj (2002) mentioned that the region located between 1,700 and $1,500 \mathrm{~cm}^{-1}$ is related to protein bands. The range 1,450 to $1,200 \mathrm{~cm}^{-1}$ is assumed to denote carboxylic groups of protein. This last region showed heritability equal to 0.34 . Picque et al. (1993) found that the MIR region located around $1,100 \mathrm{~cm}^{-1}$ corresponded to the lactose content. The wave number equal to $1,099 \mathrm{~cm}^{-1}$ had heritability equal to 0.40 , suggesting an approximation of the heritability of lactose content. Welper and Freeman (1992) estimated heritability for the percentage of lactose equal to 0.48 . Heritability of lactose content observed by Miglior et al. (2007) was 0.48 for animals in first parity. Other milk components not studied yet in current quantitative genetic research could be analyzed. For example, a study on estimation of heritability for lactate could be explored. Picque et al. (1993) mentioned that the response to lactate content was localized between 1,515 and $1,593 \mathrm{~cm}^{-1}$. The average heritability obtained for the wave numbers located in this region was 0.22 .

Besides the study of new individual milk components that are potentially interesting for breeding, the spectral data could be used directly for different purposes such as management help. As mentioned in the Introduction, the spectral data represent the entire milk composition. Moreover, the obtained results show that spectral variability is influenced by the environment. It is also known that milk composition changes when a cow has metabolic disorders such as acidosis and ketosis, or mastitis. We can, therefore, imagine using the procedure developed in this paper to study directly the changes in spectral data and therefore detect some metabolic disorders. As proposed by Mayeres et al. (2004), the information resulted from test-day model evaluations can serve to help farmers in management. Based on the difference between the expected and observed values for the spectral traits, some disorders, currently undiscovered because of the limited number of studied milk components, could be detected. In the current study, even if other, possibly finer, methods could be used for the selection of MIR regions, the possibility to strongly decrease the dimensionality of spectral traits was shown. When 8 traits are considered, use 
of common programs employed in quantitative genetics for estimating variance components or breeding values is possible.

Furthermore, the study of spectrum has a lot of potential, especially because all milk components are studied simultaneously, not just 1 or 2 specific milk components (e.g., fat and protein) without considering the other changes in milk composition. Even if more research will have to be done on the detailed meaning of spectrum, the spectral data could be used globally in the future to improve the quality of milk.

Finally, the low values observed for the permanent environmental variances ratio for the majority of traits showed that these traits reflected largely the genetic effect. Consequently, the spectral data could probably be used to reflect the effects of gene expression.

\section{CONCLUSIONS}

Considering the use of PCA to decrease the dimensionality of milk spectral traits was an interesting approach because of the high correlations existing among spectral data. The application of PCA on all spectral data showed that, based on the estimation of heritability values, not all MIR regions are of genetic interest. This observation suggests the possibility to decrease the size of spectral data analyzed for future breeding applications such as the global improvement of milk nutritional quality and the development of management tools. The MIR regions selected in the example presented in the current study suggest the possibility to consider only 8 traits. This strong decrease in the number of considered traits permits future studies related to, for instance, the detection of metabolic disorders. However, more research is required to study the observed spectral genetic variation and to find the best way to choose spectral regions and the number of considered PC for potential application.

\section{ACKNOWLEDGMENTS}

The first author acknowledges the support of the Fond pour la Recherche dans l'Industrie et l'Agriculture (Brussels, Belgium) through a grant scholarship and the National Fund for Scientific Research (Brussels, Belgium) through a grant for a scientific trip (4.552.05) and for the current position of postdoctoral researcher. Nicolas Gengler, research associate of the National Fund for Scientific Research, acknowledges the Fund's support. Additional support was provided through grants 2.4507.02F (2) and F.4552.05 of the National
Fund for Scientific Research. The authors acknowledge the technical support provided by the Walloon Breeding Association (Ciney, Belgium) and the Milk Committee (Battice, Belgium).

\section{REFERENCES}

Dufour, E., G. Mazerolles, M. F. Devaux, G. Duboz, M. H. Duployer, and N. Mouhous Riou. 2000. Phase transition of triglycerides during semi-hard cheese ripening. Int. Dairy J. 10:81-93.

Ikonen, T., K. Ahlfors, R. Kempe, M. Ojala, and O. Ruottinen. 1999. Genetic parameters for the milk coagulation properties and prevalence of noncoagulating milk in Finnish dairy cows. J. Dairy Sci. 82:205-214.

Jelen, P. 2007. Innovative uses of milk in human nutrition and health. Proceedings of the 35th Biennal Session of ICAR, Kuopio, Finland. EAAP publication 121. EAAP, Rome, Italy.

Karoui, R., A. M. Mouazen, E. Dufour, L. Pillonel, E. Schaller, D. Picque, J. De Baedemaeker, and J.-O. Bosset. 2006. A comparison and joint use of NIR and MIR spectroscopic methods for the determination of some parameters in European Emmental cheese. Eur. Food Res. Technol. 223:44-50.

Mayeres, P., J. Stoll, J. Bormann, R. Reents, and N. Gengler. 2004. Prediction of daily milk, fat, and protein production by a random regression test-day model. J. Dairy Sci. 87:1925-1933.

Miglior, F., A. Sewalem, J. Jamrozik, J. Bohmanova, D. M. Lefebvre, and R. K. Moore. 2007. Genetic analysis of milk urea nitrogen and lactose and their relationships with other production traits in Canadian Holstein cattle. J. Dairy Sci. 90:2468-2479.

Misztal, I. 1994. MTCAFS (MTC)-Multitrait REML Estimation of Variance Components Program by Canonical Transformation, With Support for Multiple Random Effects. http://nce.ads.uga. edu/ ignacy/numpub/mtc/mtcman Accessed on Sept. 23, 2008.

Misztal, I. 2008. Reliable computing in estimation of variance components. J. Anim. Breed. Genet. 125:363-370.

Misztal, I., K. Weigel, and T. J. Lawlor. 1995. Approximation of estimates of (co)variance components with multiple-trait restricted maximum likelihood by multiple diagonalization for more than one random effect. J. Dairy Sci. 78:1862-1872.

Palm, R. 1998. Notes de statistique et d'informatique: L'analyse en composantes principales: Principes et applications. Notes techniques. Faculté Universitaire des Sciences Agronomiques de Gembloux. http://www.fsagx.ac.be/si/E_index.htm Accessed on Jan. 2, 2007.

Picque, D., D. Lefier, R. Grappin, and G. Corrieu. 1993. Monitoring fermentation by infrared spectrometry: Alcoholic and lactic fermentations. Anal. Chim. Acta 279:67-72.

SAS Institute. 1994. SAS/STAT User's Guide: Version 6. SAS institute Inc., Cary, NC.

Searle, S. R., G. Casella, and C. E. McCullock. 1992. Variance Components. John Wiley \& Sons Inc., Hoboken, NJ.

Sivakesava, S., and J. Irudayaraj. 2002. Rapid determination of tetracycline in milk by FT-MIR and FT-NIR spectroscopy. J. Dairy Sci. 85:487-493.

Soyeurt, H., P. Dardenne, F. Dehareng, G. Lognay, D. Veselko, M. Marlier, C. Bertozzi, P. Mayeres, and N. Gengler. 2006. Estimating fatty acid content in cow milk using mid-infrared spectrometry. J. Dairy Sci. 89:3690-3695.

Welper, R. D., and A. E. Freeman. 1992. Genetic parameters for yields traits of Holsteins, including lactose and somatic cell score. J. Dairy Sci. 75:1342-1348.

William, P., and K. Norris. 2001. Near-Infrared Technology in the Agricultural and Food Industries. American Association of Cereal Chemists, St. Paul, MN. 